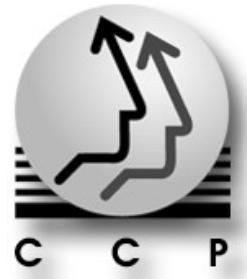

Población y Salud en Mesoamérica

Revista electrónica publicada por el

Centro Centroamericano de Población,

Universidad de Costa Rica, 2060 San José, Costa Rica

http://ccp.ucr.ac.cr

Población y Salud en Mesoamérica

Revista electrónica semestral, ISSN-1659-0201

Volumen 9, número 2, informe técnico 2

Enero - junio, 2012

Publicado 1 de enero, 2012

http://ccp.ucr.ac.cr/revista/

\title{
Estudio de relación entre Niveles de Violencia en Población masculina Privada de Libertad (PPL) en El Salvador y conocimientos de VIH, Vulnerabilidad asociada con VIH/Sida.
}

\section{Marcos Joshua Mariño Lozano}

\section{(cc) EY-NC-ND}

Protegido bajo licencia Creative Commons

Centro Centroamericano de Población 


\title{
Estudio de relación entre Niveles de Violencia en Población masculina Privada de Libertad (PPL) en EI Salvador y conocimientos de VIH, Vulnerabilidad asociada con VIH/Sida
}

\author{
Study in case of the relationship between the violence and the male population Secluded of \\ liberty (SOL) in EI Salvador and knowledge of HIV Vulnerability associated with HIV
}

\section{RESUMEN}

\author{
Marcos Joshua Mariño Lozano ${ }^{1}$
}

El presente estudio muestra, los resultados de evaluar los niveles de violencia presentes en Personas Privadas de Libertad (PPL) asociadas a la situación del VIH/Sida y su Vulnerabilidad ante la misma. Entendiendo como Vulnerabilidad el término que hace referencia a, las condiciones que una persona puede presentar en su vida que no permiten a la misma llevar actitudes correctas basadas en un cumulo de conocimientos considerado adecuado también. De aquí surge la necesidad de realizar este estudio, con el fin de determinar las herramientas necesarias para alcanzar las metas individuales en este tipo de población, así como las mismas les permitan disminuir su condición de vulnerabilidad ante la epidemia del VIH. La investigación se llevó a cabo con PPL del Centro Penal de Ciudad Barrios en San Miguel, El Salvador. Se realizó una encuesta a 175 internos donde se tomaron en cuenta variables como: edad, ocasiones que ha sido procesado en un centro penal (reincidencia), niveles de violencia, conocimientos y actitudes correctas ante el VIH/Sida Los resultados apuntan a que la mayor parte de la población es joven y se encuentran en su primer proceso como mayores de edad. Por su parte la mayoría presenta afinidad de incorporarse a un sector laboral como una manera de generar sus propios ingresos. La mayor parte de la población encuestada, muestra que a lo largo de su vida han desarrollado elevados niveles de violencia, y una gran necesidad de conocimientos correctos sobre VIH, lo que se demuestra con los altos índices de ausencia de buenas prácticas ante la epidemia-no hay un buen uso del condónfenómeno que refleja no estar sensibilizados sobre la situación.

Palabras clave: Oportunidades de cambio, personas privadas de libertad, violencia, vulnerabilidad ante el VIH/Sida

\begin{abstract}
This study shows the results of evaluating the possibilities of change, levels of violence integrated to the HIV/Aids situation. Vulnerability to HIV is a term that refers to the conditions that a person may have in his/her life that do not allow the same lead right attitudes based on a wealth of knowledge deemed appropriate as well. Thus, there is a need for People Deprived from Freedom (PPL, for its initials in Spanish) to have adequate tools to achieve their individual goals, as well as to reduce their vulnerability status towards the HIV epidemic. The investigation was conducted with PPL of the Criminal Center Ciudad Barrios in San Miguel, El Salvador. We conducted a survey of 175 interns which took into account variables such as age, times they have been prosecuted in a criminal center (recidivism), levels of violence, knowledge and right attitudes towards HIV/Aids. The results suggest that the majority of the population is young and are in their first judicial process as adults. The majority show affinity to join a labor sector as a way to generate their own income. Most of the surveyed population, shows that throughout their life have developed high levels of violence, and a great need for correct knowledge of HIV, which is demonstrated by the high rates of absence of good practices to the epidemic-there is not a good use of condoms, a phenomenon that reflects not be aware of the situation.
\end{abstract}

Keywords: Opportunities for change, persons deprived of their liberty, violence, vulnerability to HIV / Aids

Recibido: 4 abr. 2011

Aprobado: 6 oct. 2011

\footnotetext{
${ }^{1}$ Licenciado en Biología, Universidad de El Salvador. Investigador independiente. EL SALVADOR marcosjoshua@gmail.com
} 


\section{INTRODUCCIÓN}

Durante los últimos años el número de personas infectadas por el VIH /SIDA a nivel mundial, ha aumentado alarmantemente, específicamente se ha encontrado que las personas Privadas de Libertad son unos de los grupos con mayor vulnerabilidad de adquirirlo, por la presencia de múltiples conductas de riesgo. La población de personas privadas de libertad es un grupo altamente característico, que presenta situaciones que pueden facilitar condiciones de Vulnerabilidad. Este concepto será definido, como las condiciones entre conocimientos y prácticas, que no se consideren correctos frente a la transmisión del VIH. Acorde a Trepaud R. (2005), las condiciones de vida van deteriorándose, acorde a la relación que una "creciente pobreza material"; en la cual el individuo puede únicamente priorizar su misma supervivencia. La vulnerabilidad depende mucho del entorno social en los que se desarrolla un individuo, por consiguiente puede ser una gran limitante al momento de aplicar estrategias o metodologías sobre diferentes grupos sociales; he de aquí la gran importancia de elaborar estudios que relacionen ejes de distinta magnitud, con marcada relación.

La presente investigación tuvo como objetivo determinar los niveles de violencia que presentan los PPL y la correlación existente entre los conocimientos y las actitudes que se consideran correctas que los mismos presentan, frente a la epidemia del VIH.

\subsection{Privados de libertad en el Salvador (PPL)}

Privados de libertad es el término mediante el cual se conoce a toda persona, la cual ha sido despojada de su libre circulación; a causa de una condena por haber cometido un delito o se encuentra en proceso de juicio para emitir el veredicto final. Las condiciones para una persona privada de libertad, suelen ser muy especiales, ya que el hecho de tener un único entorno en el cual se desenvuelve, lo puede ubicar en condiciones desfavorables para su persona. Este factor sumado a un posible trastorno disocial, es decir aquellos patrones repetitivos y persistentes de comportamiento en el que se violan los derechos básicos de otras personas o normas sociales importantes propias de la edad.

En este caso tenemos un individuo con dos factores desfavorables presentes en su vida, por lo que su proceso de reinserción social se verá comprometido, en muchos casos puede no ser exitoso.

\subsection{Componente violencia}

La violencia es una de las problemáticas más generalizadas, no es exclusiva de ningún sistema político o económico; tiene lugar en todas las sociedades y sin distinción de posición económica, raza o cultura. La violencia tiene su origen en la discriminación y a la vez sirve para reforzarla. Las repercusiones de estas formas de violencia se extienden a familias y comunidades.

La violencia puede tener una gran cantidad de expresiones. Según J. Galtung, enfatiza la necesidad de superar la caracterización usual de la violencia o de una situación violenta: nos invita a evitar la visión tradicional sobre el tema y advierte que "habría violencia siempre que los seres humanos sean influidos para que sus realizaciones fisicas o psíquicas estén por debajo de 
sus realizaciones potenciales". En este sentido, avanzamos en la caracterización de la violencia reconociendo:

- la violencia física (ejercida sobre nuestro cuerpo) y la violencia psicológica (amenazas, formas de adoctrinamiento); se comete una violación de derechos por acción directa (los derechos de personas o grupos se ven limitados);

- las presiones que puede sufrir un ser humano; éstas influyen y/o distorsionan la posibilidad o las posibilidades reales a través de las cuales puede alcanzar su pleno desarrollo; es decir, recibe influencias — sea a través de castigos, sea a través de premios - que le impiden asumir sus decisiones, ejercer su autonomía;

- la existencia o no de un sujeto que lleva a cabo o es responsable directo de una manifestación violenta; es decir, puede existir un sujeto activo, que ejerce violencia directa, o si bien el sujeto no existe, impera la violencia estructural, tal es el caso de aquellas emergencias llamadas silenciosas, por ejemplo extrema pobreza; que impiden o menoscaban el desarrollo humano. Puede existir violencia por acción indirecta (una acción general que perjudica a un grupo que requiere tratamiento diferenciado) o por omisión (cuando hay ausencia de acciones para solucionar un problema determinado), frecuente en cuanto a los derechos sociales y económicos.

Violencia es una condición que puede marcar significativamente, el desarrollo de una persona. La violencia como una realidad posee una gran diversidad de problemáticas sobre las cuales, presenta campo de acción. En el caso de la situación actual del VIH/Sida, es un factor con una muy alta relación, en este caso nos referimos a otro tipo de violencia conocida como "Violencia de género".

La violencia de género, está directamente vinculada a la desigual distribución del poder y a las relaciones asimétricas que se establecen entre hombres y mujeres en nuestra sociedad, que perpetúan la desvalorización de lo femenino y su subordinación a lo masculino. Lo que diferencia a este tipo de violencia de otras formas de agresión y coerción es que el factor de riesgo o de vulnerabilidad es el solo hecho de ser mujer.

La violencia de género puede adoptar diversas formas, lo que permite clasificar el delito, de acuerdo con la relación en que ésta se enmarca y el ejercicio de poder que supone, en las siguientes categorías: violación sexual e incesto, asedio sexual en el trabajo y en las instituciones de educación, violencia sexual contra mujeres detenidas o presas, actos de violencia contra las mujeres desarraigadas, tráfico de mujeres y violencia doméstica. Desde esta perspectiva, es que la violencia guarda su relación tan estrecha con la transmisión del VIH, ya que imposibilita a una parte de la población en este caso la mujer, ser parte de la respuesta nacional, colocándola en una condición vulnerable.

Unos de los factores más determinantes es el hecho de que gran parte de la población desconoce sus propios derechos, para Sánchez D. (2007) la Declaración Universal de los Derechos Humanos se ha convertido en una referencia clave en el debate ético-político actual, y el lenguaje de los derechos se ha incorporado a la conciencia colectiva de muchas sociedades. Sin embargo, existe un permanente debate en el ámbito de la filosofía y las ciencias políticas sobre la naturaleza, fundamentación, contenido e incluso la existencia de los derechos humanos; y también claros 
problemas en cuanto a su eficacia, dado que existe una gran desproporción entre lo violado y lo garantizado estatalmente.

\subsection{Centros penales y VIH/Sida}

Los Centros penales desde un punto de vista estructural, son lugares donde sin importar las condiciones de aislamiento, siempre presentan determinantes sociales, políticos y culturales. Este fenómeno ha dificultado el trabajo de, gobierno, instituciones no gubernamentales, entre otros; convirtiendo estos recintos en uno de los principales problemas a resolver.

La situación del VIH/Sida en centros penitenciarios es un problema de salud pública, el cual es necesario abordar desde una perspectiva integral, donde interactúen varios componentes sociales.

El Plan Estratégico Nacional (PEN), reconoce a las personas privadas de libertad, como una población vulnerable ante la epidemia del VIH/Sida, así como uno de los grupos sociales con mayores condiciones de riesgo, presentando una mayor necesidad en la atención que se le brinde, promoviendo el desarrollo de acciones puntuales en vista a su beneficio.

Es un factor muy importante el garantizar a la población de centros penales, servicios como educación y salud, ya que los centros penitenciarios en determinado momento pueden aumentar el riesgo ante la transmisión de infecciones, entre los mismos internos, aumentando el riesgo de que estas infecciones tengan un mayor impacto, cuando los internos tienen contacto con el exterior ya sea por casos como visitas o al ser liberados.

Desde este se reconoce la importancia que posee realizar estudios, sobre todas las variables que tengan relación potencial ante esta problemática. Colaborando a disminuir la débil realización de estudios o diagnósticos que demuestren la realidad de las personas privadas de Libertad.

\subsection{Ppl como población vulnerable frente al VIH}

Bermúdez, Buela-Casal y Uribe (2005), determinaron una escala que mide conocimientos, actitudes, susceptibilidad y autoeficacia frente al VIH/sida. Los privados de libertad, hombres y mujeres, constituyen por su misma condición de cautiverio un riesgo ante el VIH. En Muchas ocasiones, se ven forzados a mantener relaciones sexuales, en su mayoría desprotegidas, dentro del centro penal y asimismo con su pareja que los visita. Esta última a su vez, podría mantener relaciones sexuales con otra pareja fuera del centro penal, estando así ambos en alto riesgo de infección o ser agentes de transmisión.

Otro factor muy importante es el desconocimiento de la naturaleza de la infección causada por el virus del VIH. En esta población, no puede tenerse grandes expectativas sobre, un desarrollo académico considerable, en muchos casos algunos/as no han logrado finalizar su educación media. Esta razón hace que los/as privados de libertad, conserven muchos mitos asociados a la transmisión del VIH. 


\subsection{Relación entre Violencia y VIH}

La teoría de Stephen R. Covey, habla de las 4 etapas fundamentales de la psicología de los humanos, donde enmarca: Supervivencia, estabilidad, logro de metas y el aporte social del individuo. Bajo una contextualización de la psicología de la pobreza, un individuo no es capaz de superar la primera etapa, por lo cual enfocara sus acciones a resolver problemáticas inmediatas sin plantearse ninguna meta específica, así mismo su aporte a la sociedad será mínimo en caso de existir. Es importante determinar que los actos violentos no surgen bajo ninguna dinámica de superación. Esta característica no permite a una persona visualizar un auto cuido, razón de suma importancia, ya que el concepto de la vulnerabilidad se basa en la misma. Conductas consideradas correctas bajo la estrategia Norteamericana del (ABC por sus siglas en inglés) donde se recomienda a la población: Abstinencia Sexual, Fidelidad y Uso del Preservativo; requieren de muchos cambios de comportamiento, los cuales denotan un interés en alcanzar una meta ya establecida. Así como algunas normas rigurosas de conducta, en las prácticas sexuales bajo el entorno ideal. Es por esta razón que al hablar de personas, que no cuentan con un Proyecto de vida, y han desarrollado altos niveles de violencia, difícilmente pueden presentar competencias para evitar la infección del VIH en sus vidas.

\section{DISEÑO METODOLÓGICO}

\subsection{Delimitación del Área de Estudio}

Ciudad Barrios es un municipio de San Miguel, ubicado en la zona oriental del país, a $160 \mathrm{~km}$ al oriente de la capital, San Salvador. El municipio tiene una extensión territorial de $68 \mathrm{Km}$ y una población estimada de 40, 500 habitantes (Figura 1). 
Figura 1: Mapa geográfico de Ciudad Barrios departamento de San Miguel, EI Salvador

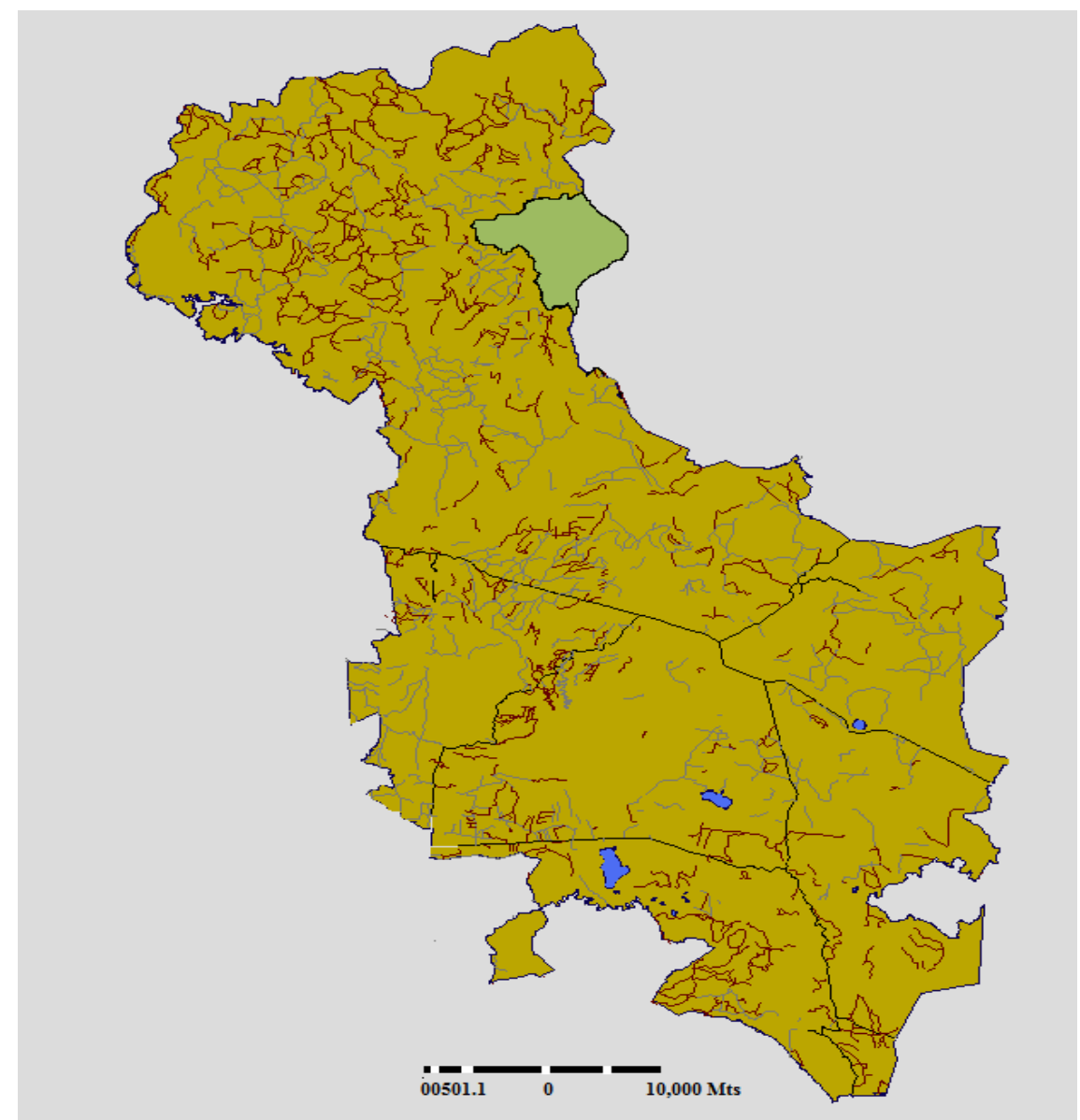

Fuente: Arc view Gis 32.

\subsection{Tipo de Estudio}

Según el tiempo de ocurrencia de los hechos y registros de la información fue prospectivo ya que la información se fue registrando según ocurrieron los fenómenos. Según el periodo y secuencia del estudio: transversal, porque las variables se estudiaron simultáneamente y en un periodo de tiempo determinado. Según el análisis y el alcance de los resultados: descriptivo, porque el propósito fue determinar los niveles de violencia de los internos en relación a sus conocimientos y actitudes consideradas correctas frente al VIH/Sida. (Day R. \& Gassel B. 2008)

Universo: Según los reportes de Lic. José Luís Rochac, Director del centro Penal de Ciudad Barrios este alberga 1750 Hombres privados de Libertad.

Muestra: Según Canales et. al (1994) La muestra debe de cumplir al menos el 10\% del universo por lo que se tiene como muestra un total de 175 Hombres privados de Libertad (PPL) del Centro Penal de Ciudad Barrios, elegidos aleatoriamente. 
Tipo de muestreo: Fue sistemático, para lo cual se utilizaron las siguientes formulas:

$\mathrm{N}=$ Universo (tamaño del universo)

$\mathrm{n}=$ Muestra (10\% del universo):

Muestra $10 \%$ de $\mathrm{N}=1750$

$1750--------100 \%$

X------------10\%

\subsection{Recolección de datos}

La recolección de datos tuvo lugar dentro de las instalaciones del penal, en el área designada para la escuela de los internos. Se realizó una jornada de 9 horas consecutivas, en conjunto con La Secretaría de La Juventud; donde tuvo lugar también una Jornada médica de consulta general. Se realizó de forma individual una encuesta cerrada a los internos, con el fin de determinar las variables de: Edad, Niveles de violencia, Conocimientos y practicas consideradas correctas frente al VIH: formas de transmisión, formas de prevención y utilización de condón en su última relación sexual.

\subsection{Operacionalización de las variables}

Tabla 1: Operacionalización de las variables involucradas

\begin{tabular}{|c|c|c|c|}
\hline Variable & Dimensión & Sub Dimensión & Indicadores \\
\hline Edad. & $\begin{array}{l}\text { Posibilidades en cambio, } \\
\text { en relación a la edad. }\end{array}$ & Demográficos. & $\begin{array}{l}\text { Jóvenes internos de centros } \\
\text { penales }\end{array}$ \\
\hline Violencia. & $\begin{array}{l}\text { Factores que han } \\
\text { imposibilitado su } \\
\text { reinserción. } \\
\text { La cantidad de veces que } \\
\text { ha estado en un centro } \\
\text { penitenciario. } \\
\text { Tipo de delito cometido }\end{array}$ & $\begin{array}{l}\text { Reincidencia. } \\
\text { Posibilidad de } \\
\text { reinserción. } \\
\text { Tipos de } \\
\text { violencia }\end{array}$ & $\begin{array}{l}\text { Número de veces que ha sido } \\
\text { procesado en su vida. } \\
\text { Tipo de delito valorado en nivel } \\
\text { de violencia: } \\
\text { - Homicidio (5) } \\
\text { - Asociaciones ilícitas (4) } \\
\text { - Violación (3) } \\
\text { - Disturbios públicos (2) } \\
\text { - Fraude o robo (1) }\end{array}$ \\
\hline $\begin{array}{l}\text { Vulnerabilidad } \\
\text { frente a la } \\
\text { infección del } \\
\text { VIH/SIDA }\end{array}$ & $\begin{array}{l}\text { Vulnerabilidad que los } \\
\text { individuos presentan a } \\
\text { adquirir la infección del } \\
\text { VIH/Sida. }\end{array}$ & $\begin{array}{l}\text { Conocimientos } \\
\text { correctos sobre } \\
\text { VIH/Sida. } \\
\text { Conductas de } \\
\text { Riesgo. }\end{array}$ & $\begin{array}{l}\text { - Conocimiento de formas } \\
\text { de transmisión } \\
\text { - Uso del condón. }\end{array}$ \\
\hline
\end{tabular}




\subsection{Técnica e instrumento}

Técnica: encuesta cerrada.

Instrumento: encuesta cerrada con la cual se obtuvo información de los componentes de Violencia, clasificados acorde a la gravedad involucrada, conocimientos y actitudes consideradas correctas frente al VIH/Sida.

\section{RESULTADOS}

Este apartado muestra los resultados porcentuales obtenidos a partir de la evaluación realizada a 175 personas privadas de libertad del Centro Penal de Ciudad Barrios. La información se sintetizo en Gráficos de proporción estadística. Se efectuó un análisis descriptivo de la información, donde se presenta el vaciado de datos obtenidos, las bases de datos que se procesaron en el paquete Epi Info 3.4 .3 (2007), para efectuar el análisis estadístico se utilizó el programa Microsoft Excel (2007).

\subsection{Edad y Reincidencia}

La mayor parte de personas participantes reflejaron estar entre los 23-27 años representando un $58.09 \%$ del total de personas encuestadas. Un $67.50 \%$ refiere haber sido procesado por un delito solo una vez en su vida.

\section{Figura 2: Porcentaje del tipo de delitos cometidos por los internos del Centro Penal de}

\section{Ciudad Barrios}

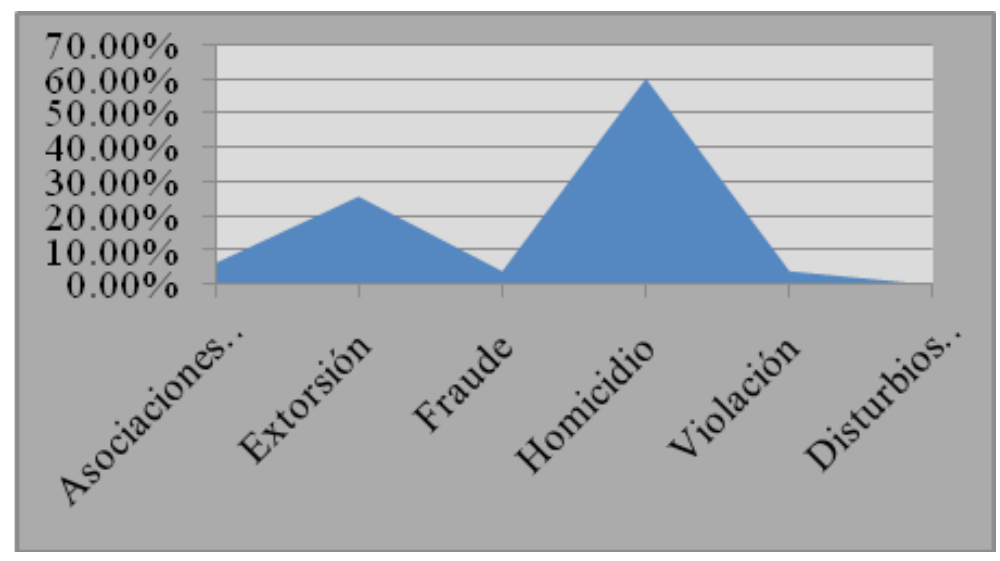

Fuente: elaboración propia

La figura 2 revela que el mayor porcentaje pertenece al delito de homicidio, el cual se reportó en un $60 \%$ de la población entrevistada. 


\subsection{Conocimiento de formas de transmisión}

Figura 3: Porcentaje de conocimiento de las formas de transmisión del VIH/Sida

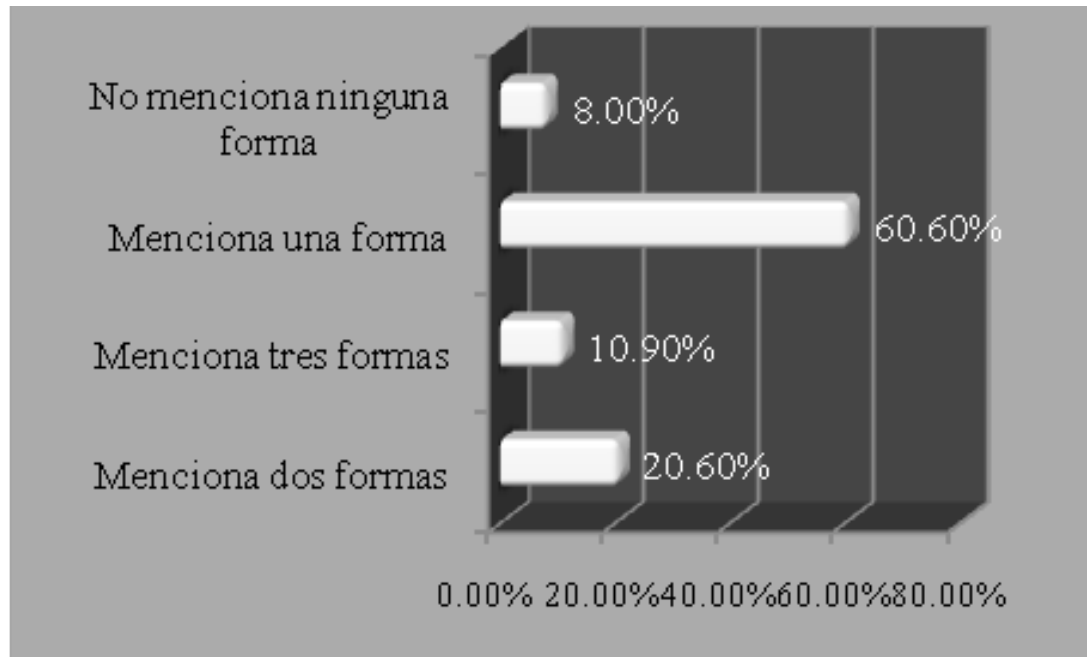

Fuente: elaboración propia

La figura 3, muestra que la mayor parte de la población sólo reconoce una forma de transmisión del VIH/Sida, con $60.06 \%$.

\subsection{Actitudes correctas ante el VIH/Sida}

Figura 4: Frecuencia de PPL que manifiestan utilizar un condón en todas sus relaciones sexuales

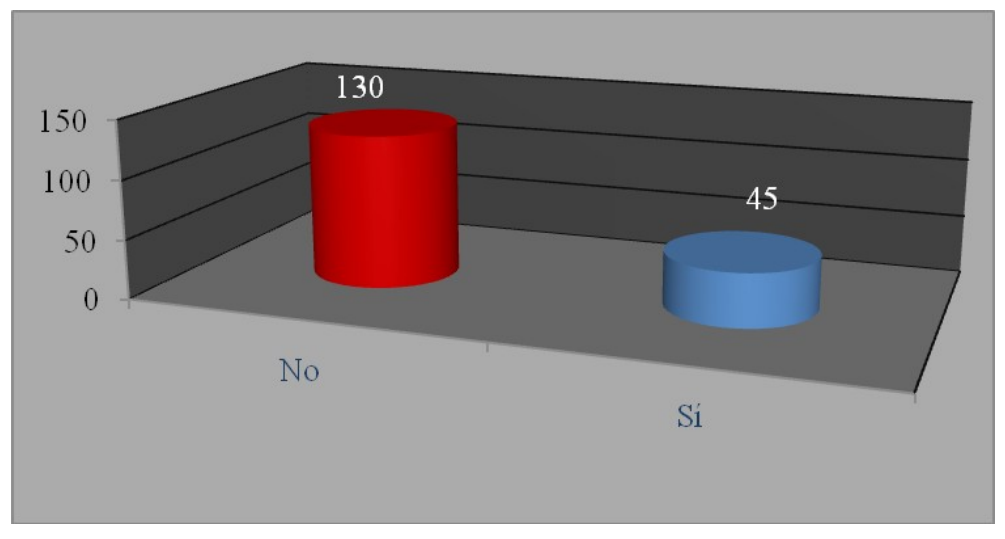

Fuente: elaboración propia

La figura 4 muestra la frecuencia de internos del centro penal de Ciudad Barrios que manifestaron tener un uso consistente del preservativo masculino. 


\section{DISCUSIÓN}

Según los resultados obtenidos, al comparar las diversas situaciones en base a la edad podemos asumir que la población de PPL son jóvenes entre los 18 y 27 años. Este resultado nos permite suponer que existe una gran ausencia de procesos de desarrollo de habilidades para la vida que, es un factor inminente que limitan a los jóvenes a incursionar en actos delictivos e integrase en pandillas juveniles.

Podemos observar que en su mayor parte los internos se encuentran en su primer proceso penal. Este factor necesita consolidarse porque cabe la posibilidad que sea su primer proceso como persona mayor de edad. Es un punto favorable en el cual existe la posibilidad de mejorar su desarrollo implementando procesos de aprendizaje.

Es un hecho relevante el que la población conformada en su mayoría por jóvenes, no pueda reconocer las formas de transmisión del VIH/Sida, así como únicamente poder reconocer una forma de prevención (ver figura 3). El hecho de estar en un rango de edad entre los 18 a 27 años, ya los ubica como una posible población vulnerable, ya que esta es la edad dónde una persona puede presentar mayor cantidad de conductas de riesgo; es un punto de considerable importancia al añadirlo a la condición de aislamiento.

La figura No. 2, indica el hecho que la población interna, posee niveles de violencia muy altos, ya que concluido por que el tipo de delito más frecuente es homicidio.

En la población se hace evidente el hecho que a medida los niveles de violencia, son más altos la población desconoce temáticas afines a la transmisión y prevención del VIH/sida. Sin embargo esta relación puede ser afectada por más causas como: Falta de interés por parte de las personas a participar en procesos educativos sobre el tema debido al entorno social en el que se desenvuelven o también puede indicar que esta población ha tenido un acceso limitado a la información necesaria.

Las actitudes correctas ante el VIH/Sida, se pueden evidenciar en base a la población que reporta tener un uso consistente del condón. Podemos evidenciar en la figura No.4, que la frecuencia es de 45 hombres los cuales manifiestan sí usar el mecanismo de barrera, mientras que la población que acepta no utilizarlo es de 130 que representa el $74.29 \%$, de la población encuestada. Los valores nos indican el hecho que la población no se encuentra sensibilizada ante la situación del $\mathrm{VIH}$, un componente muy relacionado a su falta de conocimiento sobre las formas de transmisión.

Los resultados nos muestran en general la necesidad de la población por tener mayor información sobre VIH/Sida, según la Organización Panamericana de la Salud (2007), la violencia es uno de las problemáticas que posee mayor influencia sobre la epidemia del VIH, esta aseveración puede ser sustentada en base a los resultados obtenidos, ya que los niveles de violencia en los hombres internos del penal de Ciudad Barrios, son muy altos y se evidencia que los conocimientos sobre 
VIH con actitudes correctas no resultan satisfactorios para disminuir la transmisión del VIH en la población.

\section{CONCLUSIONES}

El presente estudio, destaca la fuerte relación existente entre los niveles de violencia y vulnerabilidad al VIH. Con base a los resultados obtenidos, se concluye que en general los internos del penal de Ciudad Barrios, presentan elevados niveles de violencia, así como una gran deficiencia en temáticas sobre VIH/Sida acompañadas de prácticas no adecuadas como el sexo sin protección.

Acorde a los resultados obtenidos del presente estudio, se concluye que la violencia es creciente en poblaciones juveniles, es decir que existe un deterioro social, el cual tiene un impacto negativo sobre la percepción del proyecto de vida en los jóvenes, este conserva una estrecha relación acorde a las condiciones de bajo desarrollo social, con las cuales deben coexistir.

Acorde a los resultados se concluye que la mayor parte de PPL son personas comprendidas entre los 15 y 24 años, bajo el esquema Nacional de atención y control de la infección causada por el VIH; se crea una doble condición de vulnerabilidad, ya que según el Plan Estratégico Nacional 2005-2010, las personas en este grupo de edad son consideradas en condiciones de riesgo así mismo las PPL por las condiciones adversas y el bajo apego a un seguimiento en el alcance de sus metas.

Se concluye que las personas privadas de libertad en gran parte de su proceso de reinserción se encuentran con una diversidad de problemáticas, que resultan ser una limitante a nivel multisectorial, por lo que necesitan un abordaje de forma integral, donde los ejes temáticos aborden, salud, disminución de violencia y educación.

\section{RECOMENDACIONES}

Se recomienda a la dirección del Centro penal de Ciudad Barrios, mantener el ritmo de trabajo que actualmente tienen, permitiendo abrir los espacios necesarios para mejorar las condiciones con la población interna y mantener la colaboración con las organizaciones e instancias interesadas a trabajar en el tema de prevención de VIH-sida.

Se recomienda a las instancias Nacionales fortalecer los programas de intervención en centros penales con el fin de disminuir los niveles de violencia de las personas internas.

Se le recomienda a las $\mathrm{ONG}^{\prime}$ s que trabajan con PPL desarrollar más procesos dirigidos, a brindar mejores oportunidades de cambio enfatizados a su reinserción social, valorando la posible ventaja que represente que la mayor parte de la población se ubica entre los 18-27 años. 


\section{REFERENCIAS BIBLIOGRÁFICAS}

American Psychiatric Association (2004?). Manual diagnóstico y estadístico de los trastornos mentales DSM IV. USA: la Asociación. http://personal.telefonica.terra.es/web/psico/dsmiv.html

Amnistía Internacional (2005). Boletín de Educación en Derechos Humanos. no. 14 Argentina

Bermúdez, M. P.; Buela-Casal, G. y Uribe, A. F. (2005). Adaptación al colombiano de la escala VIH-65. Universidad de Granada

Canales, F; Pineda B. Alavarado E (1994). Metodología de la investigación. Washington: OPS

Corvey E (2000). Viviendo los 7 Hábitos Historias que Agradecen. México: Grijalbo

Day R. \& Gassel B (2008). Cómo escribir y publicar trabajos científicos. Washington: O. P.S.

Galthung J. (1976). The Basic needs aproachin human needs, Human rights, and the theories of development. Ginebra: Institute de 'Etudes du developpement

El Salvador, Ministerio de Salud Pública y Asistencia Social (2005). Plan Estratégico Nacional para la prevención, Atención y Control del VIH-SIDA e ITS, 2005-2010. San Salvador.

O.P.S. (2007). Salud en las Américas, Volumen I - regional http://www.paho.org/hia/archivosvol2/paisesesp/El\%20Salvador\%20Spanish.pdf

El Salvador, Ministerio de Salud Pública y Asistencia Social (2008). Plan de acción conjunto para el desarrollo y fortalecimiento de la intervención en salud dirigido a personas privadas de libertad ante las ITS/VIH/SIDA en los centros penitenciarios de El Salvador. San Salvador: MSPAS

Sánchez, D (2007). Repensar derechos humanos: de la anestesia a la sinestesia. Sevilla: Editorial MAD

Vargas Trepaud R (2005). Proyecto de Vida y Planteamiento Estratégico Personal. Lima, Perú: s.e. 